\title{
Gyroscope Deviation From Geodesic Motion: QUASIRESONANT OSCILLATIONS ON A CIRCULAR ORBIT
}

\author{
O. B. Karpov \\ Moscow State Mining University \\ 119991, Moscow, Russia
}

October 15, 2018

\begin{abstract}
General relativistic spin-orbit interaction gives rise to quasiresonant oscillation of the gyroscope mass center along the orbital normal. The oscillation amplitude appears to be measurable by the present-day instruments. The influence of oblateness of the field source has been investigated.
\end{abstract}

PACS: 04.80.Cc, 04.25.Nx, 95.30.Sf

\section{Introduction}

In general relativity a motion of a spinning test body (gyroscope) undergoes a spin-orbit interaction in two aspects: 1) an influence of the orbital motion on the gyroscope rotation axes orientation, 2) an influence of the gyroscope intrinsic momentum (spin) on its orbit. The first one is comparative simple when the spin parallel transport is assumed. It is admissible if a deviation from a geodesic motion is small. The Fermi-Walker transport along appointed world line is not complicated too. The parallel transport in an spherically symmetric field along a geodesic leads to a precession of the gyroscope axes known as the geodetic or de Sitter precession [1. In the field of a rotating mass the gyroscope axes undergoes the Schiff precession [2] being to verify in the Gravity Probe B experiment (see [3] for details).

In this work the second aspect of the spin-orbit interaction is considered. The orbital motion of the gyroscope is a sophisticated problem that has not been resolved in full till now even in post-Newtonian approximation. There do exist several various approaches with different results in the main approximation (for example [4, 5, 6, 7, 8, 9, 10,). The only covariant general relativistic equations of motion of the spinning test particles are well-known Papapetrou equations [5]. This equations set is incomplete and requires supplementary conditions. It is generally accepted that these conditions single out the representative point as a gyroscope mass center, but there exist diverse other opinions 9, 10, 11, 12. Besides, the Papapetrou equations or alternative ones are very complicated. Its investigation is limited usually by a general analysis and an examination of the effects is restricted as a rule by the motion of the gyroscope with a vertical spin, i.e. the gyroscope axes orthogonal the orbital plane. It is known for example that such gyroscope moves on a circular orbit with a velocity differing from one of a body without spin [13. A gyroscope with a horizontal spin goes out the geodesic plane. A quasiresonant character of the spin-orbit interaction in this case has been revealed first in works 14, 15.

In the present work the motion of the gyroscope with the horizontal spin is investigated and the general relativistic effect of quasiresonant beating is proposed. Due to a small denominator the speed of light in an oscillation amplitude is cancelled and therefore the effect turns out quite sizable. The obvious physical interpretation of the effect is given. This effect does not depend on supplementary conditions and is the same in the different approaches [4, 5, 6, 7, , 8, ,9, 10. A significant simplification of the description is achieved by the expansion of the equations of motion linear in the displacement from a geodesic. Instead of studying the intricate gyroscope orbit having not described before, the small oscillation is investigated. This oscillation gives sufficient information about the gyroscope orbit. It is shown that a Newtonian nonsphericity of the field source causes a specific effacing of the quasiresonant beating, retaining the oscillation amplitude measurable.

In the following calculations orthonormal bases are used, Greek indices run from 0 to 3, Latin indices - from 1 to 3 . Signature is $(-+++)$. 


\section{The essence of the effect}

General relativistic spin-orbit acceleration $a$ deviating a gyroscope mass center from a geodesic is of order of

$$
a \sim \epsilon \frac{S}{\lambda} g,
$$

where $\epsilon=G M / c^{2} r$ is the relativistic small parameter, $g=G M / r^{2}$ is Newtonian acceleration due to gravity, $M$ is the source mass, $S$ is the the spin of the gyroscope, $\lambda$ is its orbital momentum, $c$ is the speed of light, $G$ is the gravitational constant. The motion of the rotating body mass center essentially (in the main approximation (10) depends on the reference frame in which it has been obtained. General expression of the spin-orbit acceleration in the main post-Newtonian approximation (11) is [7, 16]

$$
\begin{aligned}
\boldsymbol{a}=3 \frac{G M}{m c^{2} r^{3}}[\boldsymbol{S} \times \boldsymbol{v} & +(2-\sigma) \hat{\boldsymbol{r}}(\boldsymbol{S}(\hat{\boldsymbol{r}} \times \boldsymbol{v})) \\
& -(1+\sigma)(\boldsymbol{v} \hat{\boldsymbol{r}})(\boldsymbol{S} \times \hat{\boldsymbol{r}})] .
\end{aligned}
$$

Parameter $\sigma$ numbers the different mass centers: $\sigma=$ 0 corresponds to the Dixon [6] and Pirani [17] conditions (the intrinsic mass center), $\sigma=1$ corresponds to the Corinaldesi-Papapetrou conditions [18] (the mass center defined in the "rest" frame in which the gyroscope moves with a velocity $\boldsymbol{v}$ ), and $\sigma=1 / 2$ leads to the results of Fock 44 and 9, 10. For a circular orbit $(\boldsymbol{v} \boldsymbol{r}=0)$ of the gyroscope with its axis lying in the orbital plane $(\boldsymbol{S}(\boldsymbol{r} \times \boldsymbol{v})=0)$, independently of the parameter $\sigma$, the spin-orbit acceleration (2) is

$$
\boldsymbol{a}=3 \frac{G M}{m c^{2} r^{3}} \boldsymbol{S} \times \boldsymbol{v} .
$$

Parallel transport of the spin vector $\mathbf{S}$ means that in process of revolution the acceleration (3) is directed along the orbital normal $\mathbf{e}_{3}$ and is periodic in time $\tau$,

$$
\boldsymbol{a}=\boldsymbol{e}_{3} \epsilon \frac{S}{\lambda} g \cos \left(\omega_{s} \tau+\beta\right) .
$$

The frequency $\omega_{s}$ differs from the orbital frequency $\omega$ because of geodetic precession $\Omega^{G}$,

$$
\Delta \omega=\omega-\omega_{s}=\Omega^{G}=\frac{3}{2} \epsilon \omega .
$$

On the other hand, the frequency of free tidal oscillation along the orbital normal is equal to the orbital frequency. It leads to an almost resonant beating with the modulation frequency (4) and maximum amplitude

$$
A=\frac{a}{2 \omega \Delta \omega}=\frac{S}{\lambda} r .
$$

Note the cancellation of the speed of light $c$ in the amplitude (5) by the relativistic small denominator
$\Delta \omega$ (4). During the time $\tau \ll\left(\Omega^{G}\right)^{-1}$ quasiresonant oscillation enhances linearly at a rate

$$
A \Delta \omega=\frac{3}{2} \epsilon \frac{S}{\lambda} v
$$

and reaches the values measurable with the presentday instruments. For example, in the case of a gyroscope of $10^{-1} \mathrm{~m}$ in dimension with $10^{-1} \mathrm{~s}$ period of intrinsic rotation in a near-Earth orbit $r \simeq 7 \cdot 10^{3}$ $\mathrm{km}$, we get the following values

$$
\epsilon \sim 10^{-10}, \frac{S}{\lambda} \sim 10^{-9}, A \Delta \omega \sim 10^{-9} \mathrm{~cm} / \text { day } .
$$

Parasitic effects of nonrelativistic origin are mutually cancelled in the symmetric relative oscillations of two gyroscopes with antiparallel spins.

\section{The calculation of the net effect}

Static, spherically symmetric gravitational field in the post- Newtonian approximation is described by the tetrad

$$
\stackrel{\check{e}}{\mu}^{\mu}=\{(1-\epsilon) c d t,(1+\epsilon) d r, r \sin \theta d \phi,-r d \theta\}
$$

which represents the rest observers in the Schwarzschild metric. "Electric" $E$ and "magnetic" $B$ parts of the Riemann tensor $\mathcal{R}$ (see, for example, [8, 16]

$$
E_{i j}=\mathcal{R}_{i 0 j 0}, \quad 2 B_{i j}=\mathcal{R}_{i o m n} \varepsilon^{m n}{ }_{j}
$$

in this frame are

$$
\begin{aligned}
\stackrel{\circ}{E}_{i j}= & n^{2} \operatorname{diag}\{-2,1,1\}, \quad \stackrel{\circ}{B}_{i j}=0, \\
& n^{2}=G M / r^{3} .
\end{aligned}
$$

Transition to the orbital frame $\boldsymbol{e}^{\nu}$ is fulfilled by the boost $\stackrel{e}{e}^{\mu}=L^{\mu}{ }_{\nu} \boldsymbol{e}^{\nu}$ in $\stackrel{\bullet}{2}_{2}$-direction. The Lorentz matrix $L$ has the standard form. Namely, the components of the 4 -velocity of the fiducial orbital motion $\phi=n t=\omega \tau$ are

where

$$
u^{\mu}=L_{0}^{\mu}=\gamma\{1,0, \beta, 0\},
$$

$$
\begin{array}{ll}
\gamma=\left(1-\beta^{2}\right)^{-1 / 2}, & \beta=v / c, \\
v=(1+\epsilon) n r, & \omega=\gamma v / r=n(1+3 \epsilon / 2),
\end{array}
$$

and $\tau$ is the proper time. The $\boldsymbol{e}_{1}$ axis is directed along the current radius-vector, the $\boldsymbol{e}_{2}$ axis is along the orbital motion velocity, and $\boldsymbol{e}_{3}$ is orthogonal to the orbital plane:

$$
L_{1}^{1}=1, \quad L_{2}^{2}=\gamma, \quad L^{3}{ }_{3}=1 .
$$


The angular velocity vector $\boldsymbol{\Omega}$ of rotation of the orbital triad $\nabla_{u} \boldsymbol{e}^{i}=\Omega^{i}{ }_{k} \boldsymbol{e}^{k}$ has the only component

$$
\Omega_{3}=\Omega_{12} \stackrel{\text { def }}{=} \omega_{s}=n .
$$

The transformation of the "magnetic" matrix [16]

$$
\begin{aligned}
B_{i j}= & 4 \stackrel{\circ}{B}_{k l} L^{[k}{ }_{i} u^{0]} L^{[l}{ }_{j} u^{0]} \\
& -\stackrel{\circ}{B}_{p q} \varepsilon^{p}{ }_{k m} \varepsilon^{q}{ }_{l n} L^{k}{ }_{i} u^{m} L^{l}{ }_{j} u^{n} \\
& -4 \stackrel{\circ}{E}_{k m} \varepsilon^{m}{ }_{l n} L^{[k}{ }_{(i} u^{0]} L^{l}{ }_{j}{ }_{j} u^{n}
\end{aligned}
$$

leads to an appearance in orbital frame of the component

$$
B_{31}=\beta\left(\stackrel{\circ}{E}_{33}-\stackrel{\circ}{E}_{11}\right)=3 n^{2} \beta .
$$

The transformation of the "electric" matrix is analogous to (12) with a substitution $B \rightarrow E, E \rightarrow-B$ (see [16]). The result is

$$
\begin{gathered}
E_{11}=-2 n^{2}(1+\epsilon / 2), \quad E_{22}=n^{2}, \\
E_{33}=n^{2}(1+3 \epsilon)=\omega^{2} .
\end{gathered}
$$

Note the invariance of the component $E_{22}$ parallel to the boost and the equality $E_{33}=\omega^{2}$ being exact.

The equation of motion of the gyroscope mass center in the orbital frame is the equation of geodesic deviation with the spin-orbit acceleration (2) in its right-hand side $^{1}$,

$$
\nabla_{u} \nabla_{u} \xi^{i}+E_{k}^{i} \xi^{k}=a^{i}
$$

where

$$
\nabla_{u} \nabla_{u} \boldsymbol{\xi}=\ddot{\boldsymbol{\xi}}+2 \boldsymbol{\Omega} \times \dot{\boldsymbol{\xi}}+\dot{\boldsymbol{\Omega}} \times \boldsymbol{\xi}+\boldsymbol{\Omega} \times(\boldsymbol{\Omega} \times \boldsymbol{\xi}) .
$$

The dot designates the derivative with respect to proper time $\tau$. In the post-Newtonian approximation the spin-orbit force applied to the intrinsic mass center of the rotating body is

$$
m a^{i}=-c^{-1} B_{k}^{i} S^{k}
$$

This formula can be obtained, for example, by the matched asymptotic expansions method 8 or directly from the Papapetrou equations with the supplementary conditions of Pirani or Dixon (see 16] the distinctions between the exact conditions of Pirani and Dixon, ibid.).

In the equation (15) $E_{i k}$ is measured on the fiducial geodesic $u$, but $B_{i k}$ in (16) ought to be calculated in the frame comoving with the gyroscope mass

\footnotetext{
${ }^{1}$ The equation (15) can be obtained by an expansion of the Papapetrou equations linear in the displacement $\xi$ in the main approximation (1) of the spin-orbit interaction. At $\boldsymbol{S}=0 \Rightarrow$ $\boldsymbol{a}=0$ the equation 15 is reduced to the geodesic deviation equation.
}

center. This "mixing" is admissible in the approximation linear in $S$ (11) and linear in $\xi$ (15) if the displacement $\xi$ is induced by the spin-orbit interaction: $\xi \propto S, \xi S \propto S^{2} \propto \xi^{2}=0$. On the same ground we transport the spin vector according to Fermi-Walker along the fiducial geodesic,

$$
\begin{gathered}
\nabla_{u} \boldsymbol{S}=\dot{\boldsymbol{S}}+\boldsymbol{\Omega} \times \boldsymbol{S}=0 \\
\dot{S}_{1}=\omega_{s} S_{2}, \dot{S}_{2}=-\omega_{s} S_{1}, \dot{S}_{3}=0 .
\end{gathered}
$$

The parallel transport equation (17) describes the known geodetic precession (4):

$$
S_{1}=S \cos \left(\omega_{s} \tau+\beta\right), S_{2}=-S \sin \left(\omega_{s} \tau+\beta\right) .
$$

For the spin in the fiducial plane $\left(S_{3}=0\right)$, the equations of the mass center motion (15), (16) are:

$$
\left.\begin{array}{c}
\ddot{\xi}_{1}-2 \omega_{s} \dot{\xi}_{2}+\left(E_{11}-\omega_{s}^{2}\right) \xi_{1}=0, \\
\ddot{\xi}_{2}+2 \omega_{s} \dot{\xi}_{1}=0, \\
\ddot{\xi}_{3}+E_{33} \xi_{3}=3 g \epsilon \frac{S_{1}}{\lambda} .
\end{array}\right\}
$$

The equations (19) describes the free oscillation with the frequency $\omega^{\prime}=\sqrt{E_{11}-3 \omega_{s}^{2}}=n(1-3 \epsilon / 2)$, induced by an initial perturbation in the fiducial plane. The difference of $\omega^{\prime}$ from the orbital frequency $\omega$ is caused by the general relativistic pericenter drift of the perturbed quasielliptic orbit, $\omega-\omega^{\prime}=3 \epsilon n$. If the initial perturbation in the fiducial plane is zero, the trajectory of gyroscope projection onto the plane coincides with the circular geodesic.

The equation of forced oscillation (20) along the orbital normal

$$
\ddot{\xi}_{3}+\omega^{2} \xi_{3}=3 \epsilon \frac{S}{\lambda} g \cos \left(\omega_{s}+\beta\right)
$$

proves to be quasiresonant due to proximity of the frequencies of natural tidal oscillation $\sqrt{E_{33}}=\omega$ and of the compelling force $\omega_{s}$. The difference of the frequencies $\Delta \omega$ (4) preventing the oscillation from resonance is equal to the geodetic precession $\Omega^{G}$. The general solution of the equation (21)

$$
\xi_{3}=A \cos \zeta-C \cos \eta, \zeta=\omega_{s} \tau+\beta, \eta=\omega \tau+\alpha
$$

contains the amplitude $A$ (5) and two integration constants, $C$ and $\alpha$. If $C=0$, the oscillation (22) describes the precession of the gyroscope orbit, inclined by the angle $A / r=S / \lambda$ relative to the fiducial plane, with the angular velocity of the geodetic precession (4) (Fig. 1 (a)). The evolution of the gyroscope orbital momentum with arbitrary $C$ is presented on the Fig. 1 (b). If $C=A$, the pure beating occurs,

$$
\xi_{3}=2 A \sin \frac{\eta-\zeta}{2} \sin \frac{\eta+\zeta}{2} .
$$




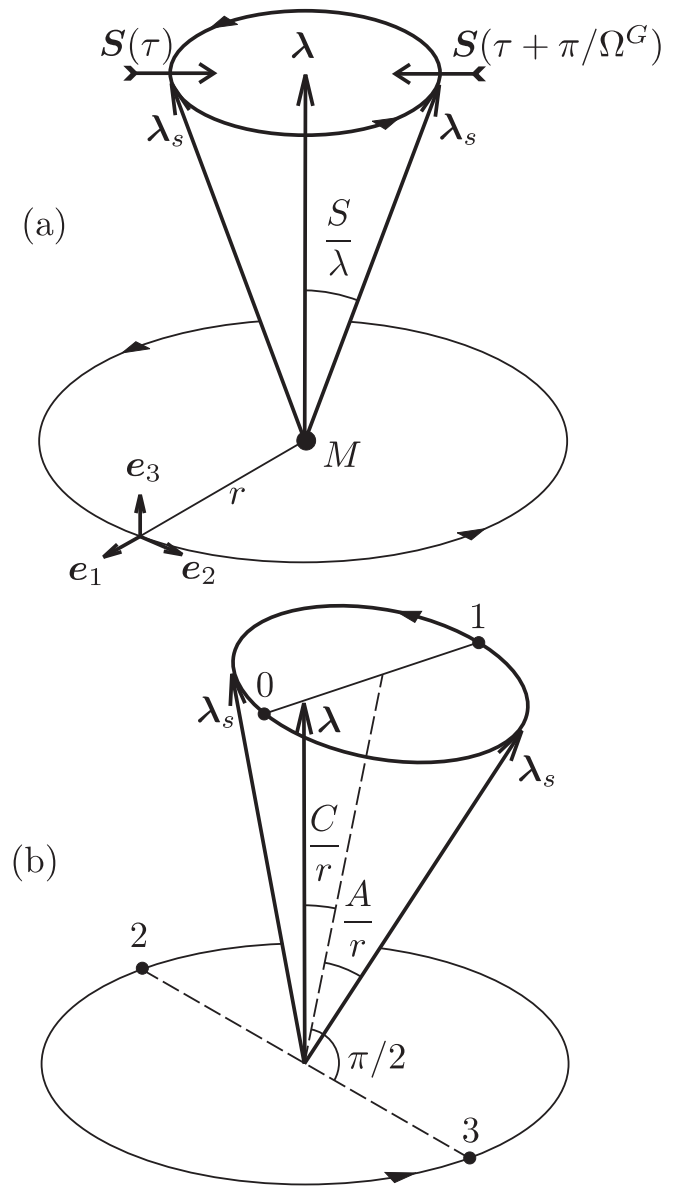

Figure 1: Orbit of the gyroscope. Orbital momenta of the fiducial geodesic and the gyroscope are $\boldsymbol{\lambda}$ and $\boldsymbol{\lambda}_{s}$ respectively. (a) Precession of the gyroscope orbit at $C=0$. (b) Variable inclination of the gyroscope orbit, the constant $C$ is arbitrary. The orbital momentum $\boldsymbol{\lambda}_{s}$ gets in the marked positions 0 and 1 when the value $\sin ((\eta-\zeta) / 2)$ equals 0 and 1 respectively. In the points 2 and 3 it turns out $\cos \eta=0$.

The mass center makes the oscillation along the orbital normal with the variable amplitude modulated by the geodetic precession (4). The initial condition $\xi_{3}(\tau=0)=0$ is provided by the choice of the constant $\alpha=-\beta$,

$$
\xi_{3}=2 A \sin \frac{\Delta \omega}{2} \tau \sin \left(\frac{\omega+\omega_{s}}{2} \tau+\beta\right)
$$

and within a time $\tau \ll(\Delta \omega)^{-1}$ the oscillation amplitude grows at a rate $A \Delta \omega$ (6), (7). The condition $\dot{\xi}_{3}(\tau=0)=0$ fixes the initial spin orientation $\sin \beta=0$ along the radial direction (see (18)).

The problem of measuring the oscillation (24) is complicated by the circumstance that the initial perturbations lead to natural tidal oscillation with the orbital frequency $\omega^{2}=E_{33}$ (see (21)). Therefore the gyroscopes with antiparallel spins must be manufactured to be coaxial. In order that the Newtonian harmonic oscillation due to instrumental error be smaller than the relativistic oscillation induced by the spinorbit interaction, the strong restrictions on the initial perturbations $\xi_{3}(0), \dot{\xi}_{3}(0)$ are needed:

$$
\xi_{3}(0) \ll \xi \sim A \Delta \omega \tau_{f}, \quad \dot{\xi}_{3}(0) \ll \omega \xi,
$$

where $\tau_{f}$ is the time of forming of the amplitude measured.

\section{Effect of field oblateness}

The Newtonian oblateness of the source does not lead to the gyroscope forced oscillation. The oblateness affects the natural tidal oscillation frequency $\left(\tilde{E}_{33}\right)^{1 / 2}$, orbital frequency $\tilde{\omega}$ and, consequently, the angular velocity $\tilde{\omega}_{s}$ of the spin rotation relative to orbital triad. The two frequencies, $\left(\tilde{E}_{33}\right)^{1 / 2}$ and $\tilde{\omega}_{s}$, enter the equation of motion of the gyroscope mass center:

$$
\ddot{\tilde{\xi}}_{3}+\tilde{E}_{33} \tilde{\xi}_{3}=3 \epsilon \frac{S}{\lambda} g \cos \left(\tilde{\omega}_{s} \tau+\beta\right) .
$$

Considering only the quadrupole moment $J_{2}$ (Earth's $J_{2} \simeq 1 \cdot 10^{-3}$ ), for an equatorial orbit we get

$$
\begin{aligned}
\sqrt{\tilde{E}_{33}} & =\omega\left(1+\frac{9}{4} J_{2} \frac{R^{2}}{r^{2}}\right), \\
\tilde{\omega} & =\omega\left(1+\frac{3}{4} J_{2} \frac{R^{2}}{r^{2}}\right), \\
\tilde{\omega}_{s} & =\omega_{s}\left(1+\frac{3}{4} J_{2} \frac{R^{2}}{r^{2}}\right),
\end{aligned}
$$

where $R$ is the equatorial radius of the source. The frequency $\left(\tilde{E}_{33}\right)^{1 / 2}$ differs from the orbital frequency $\tilde{\omega}$ because of the Newtonian quadrupole precession $\Omega^{J}$ of orbital plane,

$$
\tilde{\omega}-\sqrt{\tilde{E}_{33}}=-\frac{3}{2} \omega J_{2} \frac{R^{2}}{r^{2}}=\Omega^{J} .
$$

The gyroscope axis does not undergo the additional Newtonian precession, $\tilde{\omega}-\tilde{\omega}_{s}=\Omega^{G}$. As a result of the difference (30), the small denominator (4) is changed

$$
\begin{aligned}
\Delta \tilde{\omega} & =\sqrt{\tilde{E}_{33}}-\tilde{\omega}_{s}=\Omega^{G}-\Omega^{J} \cong-\Omega^{J} \\
& \cong \Delta \omega \frac{J_{2}}{\epsilon} \frac{R^{2}}{r^{2}}
\end{aligned}
$$

as well as the modulation period $\tilde{T}=2 \pi / \Delta \tilde{\omega}$ and the amplitude (5)

$$
\tilde{A}=-A \frac{\Omega^{G}}{\Omega^{J}}=\frac{S}{\lambda} \frac{\epsilon}{J_{2}} \frac{r^{2}}{R^{2}} .
$$


The gyroscope orbital momentum vector describes a conic surface with the apex angle $2 \tilde{A} / r$ and the time period $\tilde{T}$. The quadrupole precession period $\tilde{T}$ of a near-Earth orbit is 2 months. For the pure beating

$$
\tilde{\xi}_{3}=2 \tilde{A} \sin \frac{\Delta \tilde{\omega}}{2} \tau \sin \left[\left(\tilde{\omega}_{s}+\frac{\Delta \tilde{\omega}}{2}\right) \tau+\beta\right]
$$

within the timescale $\tau \ll \tilde{T}$ the oscillation increases just as in the case of a spherically symmetric field (6)

$$
\tilde{A} \Delta \tilde{\omega}=A \Delta \omega=\frac{3}{2} \epsilon \frac{S}{\lambda} v .
$$

The maximum amplitude formed in time $\tilde{T} / 2$ on a near-Earth orbit for the gyroscope $S / \lambda \sim 10^{-9}$ (7)

$$
\tilde{A} \sim 10^{-7} \mathrm{~cm}
$$

is several orders as good as the present-day limit of measuring small oscillations.

\section{Conclusions}

General relativistic quasiresonant spin-orbit interaction leads to the oscillation of the gyroscope mass center relative to the fiducial geodesic along the orbital normal. The beating amplitude does not include the speed of light and equals the ratio of the intrinsic momentum of the gyroscope to its orbital momentum. The modulation frequency equals the angular velocity of the geodetic precession. The oscillation represents the precession of the gyroscope orbital momentum. Within an acceptable time the oscillation amplitude reaches the values that are amenable to being analyzed experimentally.

Taking into account the source oblateness decreases the beating amplitude and increases the modulation frequency by the factor that is equal to the ratio of the quadrupole precession velocity to the geodetic precession velocity. The period of the quadrupole precession turns out to be a quite sufficient time to form a measurable amplitude of the oscillation. The tidal acceleration, providing the quasiresonant character of the oscillation, imposes strong restrictions on the initial perturbations to distinguishing the relativistic spin-orbit oscillation on the background of the Newtonian tidal oscillation.

\section{References}

[1] W. de Sitter, Mon. Not. Roy. Astron. Soc. 77, 155 (1916).

[2] L.I. Schiff, Phys. Rev. Lett. 4, 215 (1960).

[3] R.J. Adler, A.S. Silbergeit, Int. J. Theor. Phys. 39, 1291 (2000).

[4] V.A. Fock, The Theory of Space, Time and Gravitation, Mac Millan, New York (1964), §77.

[5] A. Papapetrou, Proc. Roy. Soc. Lond. A209, 248 (1951).

[6] W.G. Dixon, Nuovo Cim. 34, 317 (1964).

[7] B.M. Barker, R.F. O'Connel, J. Math. Phys. 28, 661 (1987).

[8] K.S. Thorne, J.B. Hartle, Phys. Rev. D31, 1815 (1985).

[9] A.A. Pomeransky, I.B. Khriplovich, J. Exp. Theor. Phys. 86, 839 (1998); Zh. Eksp. Teor. Fiz. 113, 1537 (1998); gr-qs/9710098

[10] A.A. Pomeransky, R.A. Sen'kov, I.B. Khriplovich, Uspekhi Fisicheskikh Nauk 170, 1129 (2000).

[11] R.I. Khapko, J. Exp. Theor. Phys. 63, 231 (1986); Zh. Eksp. Teor. Fiz. 90, 401 (1986).

[12] K. Svirskas, K. Pyragas, A. Lozdiene, Astrophys. Space Sci. 149, 39 (1988).

[13] E.N. Epikhin, N.V. Mitskievich, Izv. Vuzov. Fis. N 5, 115 (1979).

[14] O.B. Karpov, Izv. Vuzov. Fis. N 11, 68 (1982).

[15] O.B. Karpov, In: Abstr. 10th Int. Conf. Gen. Relat. Grav., Padova. 2, 965 (1983).

[16] O.B. Karpov, Preprint Moscow Eng. Phys. Inst. N 012-90 (1990).

[17] F. Pirani, Acta Phys. Polon. 15, 389 (1956).

[18] E. Corinaldesi, A. Papapetrou, Proc. Roy. Soc. Lond. A209, 259 (1959). 